\title{
Challenges of the application of data-driven models for the real-time optimization of an industrial air separation plant
}

\author{
Dionysios P. Xenos ${ }^{1, *}$, Olaf Kahrs ${ }^{2}$, Matteo Cicciotti ${ }^{3}$, Fernando Moreno Leira $^{4}$ and Nina F. Thornhill ${ }^{1}$
}

\begin{abstract}
The optimization of the operation of chemical plants may require the development of mathematical models of the process units of a plant. These mathematical models can be either first-principles or data-driven models. The former type of modeling may be complex for the use in optimization and especially for online applications such as real time optimization. Available measured process data can be used to develop the latter type of modeling. Although data-driven models offer several benefits for online applications, there are some very significant challenges related to their development in a practical industrial implementation. This paper discusses the important aspects of the building of data-driven models and demonstrates the effects of these types of models on the optimization results. The current work demonstrates the application of a real time optimization framework applied to an industrial air compressor station of an air separation plant when the models are based on operating data.
\end{abstract}

\section{INTRODUCTION}

In the last years, there has been much interest in reducing energy consumption of large industrial plants. Environmental awareness, government legislation, increased competition among companies and increasing energy prices are the major reasons that industrial plants need to improve their efficiency and operate in a more optimal way.

Optimization tools can help the users of a plant to reduce operational costs without purchasing new equipment which increases further the capital cost of the initial design. These tools rely on mathematical models which represent the behaviour of the process units of the plant. The mathematical models can be derived usually either from first-principles models or models based on raw data from the operation. The latter types of models are known as data-driven, blackbox, surrogate or meta models. Although a data-driven model does not have any physical meaning, it can be suitable for online optimization applications as this type of model offers several benefits compared to a large and complex firstprinciples model, for example reduced computational time to find solution and ease of development. .

Available process measurements of the plant can be used to develop computationally cheap models tailored to optimization frameworks. However, having installed adequate and accurate online sensors is one of the major obstacles

1 Department of Chemical Engineering, Centre for Process Systems Engineering, Imperial College London, London SW7 2AZ, UK *d. xenosaimperial.ac.uk

${ }^{2}$ Corporate Operational Excellence - Technical Process Optimisation, BASF SE, 67056 Ludwigshafen, Germany

${ }^{3}$ Process Control Task Force, BASF SE, 67056 Ludwigshafen, Germany

${ }^{4}$ Automation Technology - Advanced Process Control, BASF SE, 67056 Ludwigshafen, Germany in the process industry, especially for older machines. Each technical upgrade needs to be justified by safety conditions or improved economics. This paper discusses the challenges of the use of data-driven models for industrial applications and presents results from an example of the optimization of a network of compressors of an air separation plant.

\section{OPTIMIZATION OF COMPRESSOR STATIONS}

Compressor stations include a network of compressors operating in parallel and can be found in process systems [1], [2], in natural gas boosting stations [3] and in conventional natural gas transportation stations [4]. Han et al. [2] reported that the energy consumed by the air- and gas-network of the compressors of a terepthalic acid manufacturing plant is estimated approximately $75 \%-85 \%$ of the total consumption. Air compressors of air separation plants can consume more than $70 \%$ of the overall consumption of plants of several tens of MW [1].

The optimization of the operation of compressor stations can lead to significant reduction in energy cost compared to the typical industrial operation with strategies such as equal split and equal surge margin. A summary of typical load sharing techniques and other best practices can be seen in the article by Xenos et al. [1]. The optimization of the operation of the compressors exploits the fact that it is difficult or impossible for different compressors of a compressor station to have identical characteristics and efficiencies [5], [6]. Moreover, these characteristics and efficiencies change over time due to fouling and erosion, and non-uniform maintenance plans which result in dissimilar compressor maps for the same compressor at different time periods [3], [7].

\section{ONLINE OPTIMIZATION FRAMEWORK}

Paparella et al. [3] and Xenos et. al. [1] presented online optimization frameworks for minimizing the power consumption of compressors in real time. The basic components of a Real Time Optimization (RTO) application of industrial multi-stage centrifugal compressors can be seen in article [1]. The sensors of the monitoring system collect process data of the operation such as mass flow rates, pressures and temperatures. A steady-state identification algorithm examines key process variables and identifies when the operation is in steady-state. Thus, if the operation is in steady-state, then the collected data should be validated. The next step is to use the validated data to update the models of the online compressors (parameter estimation). An optimization model employs these data-driven models and estimates the set points of the controlled variables for optimal load sharing. 
These variables are the mass flow rates of the compressors. The set points are given to the control system which role is to apply and keep these points to the process until the next run of the RTO.

Companies such as Petrotech [6] and Siemens [8] have developed commercial tools to deal with the load sharing of the compressors but focusing on the optimization of the distance between operating points and operational safety boundaries. However, optimal load sharing for different operational conditions can be achieved with the estimation of the performances or power consumptions of the individual compressors and the use of optimization in real time. One method to estimate the performance or power consumption is with the use of available process data.

\section{DESCRIPTION OF INDUSTRIAL CASE}

\section{A. Network of parallel compressors}

The paper considers the real time optimization of an industrial case study which involves a network of parallel multi-stage centrifugal air compressors which distribute compressed air to different users such as air separation columns and other process plants of the whole site. Figure 1 shows that the industrial case study will focus on the optimization of three parallel compressors $i \in\{i 1, i 2, i 3\}$, which are part of the whole network, providing compressed air to an air separation column (i.e. downstream process).

A description of the implemented control structure in the industrial plant can be seen in Fig. 1a. The set point of the demand $M_{d e m}$ is given as an input of the system to controller FC4. The controller FC4 gives the same angle of the inlet guide vanes (IGVs) to each compressor in order to control their mass flow rates and meet the requested demand. By applying this control strategy the load should be equally shared among the compressors. If there is a mismatch between the total flow measured from FT4 and the given set point $M_{\text {dem }}$ (demand), then the IGVs of all the compressors are adjusted uniformly to reach the desired $M_{d e m}$.

By contrast, the control strategy that is required for optimal load sharing with the use of RTO can be seen in Fig. 1b. The three flow transmitters FT1, FT2 and FT3 are used to measure the individual flows at the exit of the compressors. The RTO receives the desired $M_{d e m}$ as input and the FT4 measures the total mass flow rate of the compressors. In this case controllers FC1, FC2 and FC3 work independently and they receive the values of the flows of the compressors according to the RTO computations. The RTO estimates the best set points for FC1, FC2 and FC3 based also on measurements of $T_{i n}, P_{i n}$ and $P_{o p}$. The individual feedback controllers FC1-FC3 have to independently adjust the position of the IGVs of the compressors to reach the set points of the mass flows given from the RTO.

\section{B. Description of multi-stage compressor}

The schematic of a compressor of the industrial case study can be seen in Fig. 2. The compressor is centrifugal multistage, with a cooling system, driven by electrical motors with constant rotational speed. The major available measurements

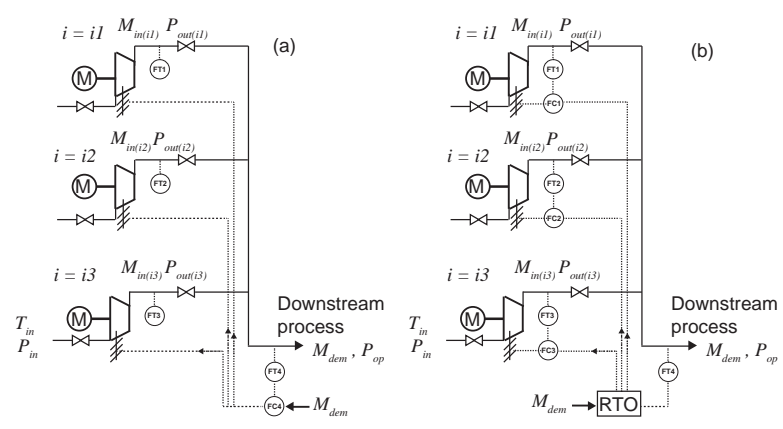

Fig. 1. The current implemented control scheme of the industrial case (a). The proposed control scheme (b) includes the Real Time Optimization (RTO) block.

which can be provided online from the DCS and will be used to create data-driven models can be seen in the list:

- inlet mass flow rate $M_{i n}$ in $\mathrm{kg} / \mathrm{s}$

- inlet pressure $P_{i n}$ in bar

- inlet temperature $T_{i n}$ in $\mathrm{K}$

- outlet pressure (after the aftercooler) $P_{\text {out }}$ in bar

- power consumption $W_{e l}$ in $\mathrm{kW}$

There are also other measurements available which are not presented in the list, for example the humidity of the ambient air, the outlet temperature (after the aftercooler) and the angle of the inlet guide vanes. These measurements are not listed as they are not used in the developed data-driven models [1]. For most of the compressors of the case study, there are not available measurements between the stages, for example temperatures and pressures at the discharge of the intermediate stages. Only one compressor includes measurements between the stages. The compressor station involves more than ten installed compressors, however only two or three compressors are usually used for serving the same downstream process, as shown in Fig. 1.

\section{TOPICS RELATED TO IMPLEMENTATION}

\section{A. Availability of data and model development}

The availability of field measurements is a factor which influences the selection of a suitable model of a process unit. The thermodynamic equation for the calculation of the isentropic efficiency of a single-stage compressor $\eta_{i s}=$ $W_{i s} / W_{\text {actual }}$ has to estimate the isentropic power (1) and the actual power (2). The $c_{p}$ is the specific heat capacity of the fluid and the $\gamma$ is the isentropic exponent. These expressions are valid for a single-stage compressor or for a the case of multi-stage compressor with the assumptions that

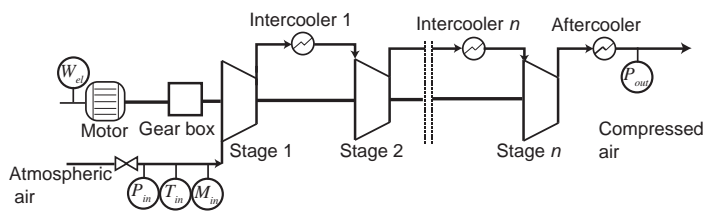

Fig. 2. An industrial multi-stage air compressor. 
the temperature and pressure at the exit of each intercooler, and at the inlet of the stage after the intercooler are the same as the temperature and pressure at the inlet of the previous stage.

$$
\begin{gathered}
W_{\text {is }}=M_{\text {in }} c_{p} T_{\text {in }}\left({\frac{P_{\text {out }}}{P_{\text {in }}}}^{\frac{\gamma-1}{\gamma}}-1\right) \\
W_{\text {act }}=M_{\text {in }} c_{p}\left(T_{\text {out }}-T_{\text {in }}\right)
\end{gathered}
$$

In the case of the multi-stage compressor of the industrial case, the geometries of the stages of each compressor are different and the sizes of the various intercoolers are not the same. Because of the lack of measurements between the stages, thermodynamic equations cannot not be used for developing model for each compressor stage and intercooler. According to Fig. 2, the $P_{\text {out }}$ of each individual stage is not available and therefore the discharge pressure cannot be used in Eq. (1). Also, the outlet temperature of each stage is not available, therefore the actual power (2) used by each compressor stage cannot be estimated. There are no measurements for mass flow rates, temperatures and pressures related to the intercoolers.

The efficiency of the electrical motor and the losses in the gearbox should be considered for calculating the total efficiency of the multi-stage compressor. Another issue with the availability of measurements is that there are measurements which may be available for some periods but their availability is not consistent due to failures in the sensor system and due to the time needed to replace a failed sensor.

As there is not enough information for developing firstprinciples models for all the compressors, models have to be built on the basis of those process measurements which are readily available such as inlet temperature, inlet pressure and outlet pressure. These measurements are sufficient to support a data-driven model which can express the actual power of the motor $W_{e l}^{a c t}$ of each compressor as a function $f$ of these basic process variables including a model error.

$$
W_{e l}^{a c t}=f\left(\mathbf{z}, M_{i n}, \mathbf{b}\right)+\text { model error }
$$

The vector $\mathbf{z}$ includes the parameters such as inlet temperature and pressure, the vector $\mathbf{b}$ involves the coefficients of the function $f$ which are estimated through regression [1], and the $M_{i n}$ is the mass flow rate of the compressor. The mass flow rate of the compressor is the decision variable in the optimization problem which can be modified with the use of the inlet guide vanes. The function $f$ represents the predicted power consumption $W_{e l}^{\text {pred }}$.

The decision of which variables should be used for the development of a data-driven model is not a standard procedure. Analysis of the raw data using observation or statistical methods such as the covariance matrices [9] is the first step of the building of the models. Figure 3 shows the evolution of the power consumption of the motor of compressor $i 1$, its mass flow rate, outlet pressure and inlet temperature. From the observation of the figure, it is obvious that the mass flow rate significantly influences the power of the motor. This was confirmed by the covariance matrix which showed the

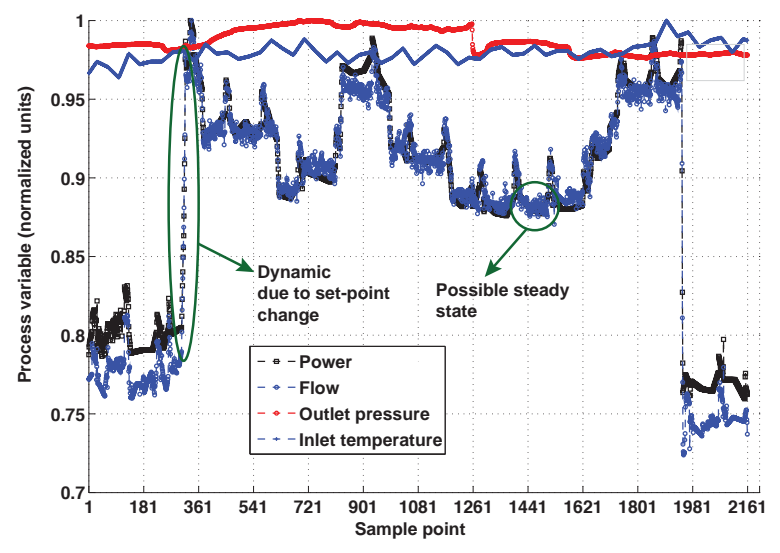

Fig. 3. Evolution of process variables over time.

power consumption and mass flow rate were significantly correlated.

\section{B. Uncertainty in optimization results due to model error}

The objective of the RTO is to estimate the key variables of the operation of the compressors which result in reduced total power consumption while at the same time ensuring that all the constraints related to the compressors and plant are satisfied. The formulation of the optimization problem of the RTO has been presented in article [1]. The objective function of the optimization is the minimization of the total predicted power consumption:

$$
\min _{M_{i n(i)}} \sum_{i \in I} W_{e l(i)}^{\text {pred }}
$$

The power consumption $W_{\text {el }(i)}^{\text {pred }}$ of each compressor $i$ has a prediction error $\Delta W_{e l(i)}$. The root square mean error (RSME) [1] is used to model the prediction error for each power consumption.

$$
\Delta W_{e l(i)}=\sqrt{\sum_{k=1}^{k=N}\left(W_{(i, k)}^{a c t}-W_{(i, k)}^{\text {pred }}\right)^{2} / N}
$$

where $k=1,2, \ldots, N$ is the number of the observations of the data set of the power consumption.

As the objective function is the summation of the power consumptions of the compressors, then the uncertainty (i.e. error) $\Delta W_{e l, t o t}$ of the objective function (4) can be estimated by the following equation:

$$
\Delta W_{e l, t o t}=\sqrt{\sum_{i \in I} \Delta W_{e l(i)}^{2}}
$$

The application of the real time optimization based on paper [1] identified fifty steady states in a $12 \mathrm{~h}$ operation of the compressors shown in Fig. 1. The models of the power consumptions were developed with historical data of 15 days. This section now proceeds to give an analysis and commentary on the impact of modelling errors on the 


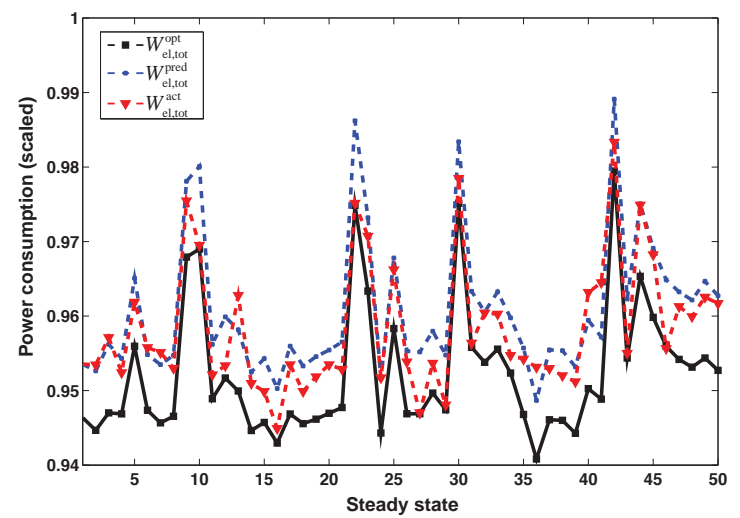

Fig. 4. Total power consumption of the three compressors for each each steady state.

optimization results. The uncertainty of the model for each compressor is equal to the RMSE value estimated in [1].

Figure 4 shows the optimal power $W_{e l, t o t}^{\text {opt }}$ of the three compressors $i 1, i 2$ and $i 3$ estimated by an optimization model [1] and the predicted power consumption $W_{\text {el,tot }}^{\text {pred }}$ based on the same data-driven models used in the optimization, but the set points of the operators were used in the models. The actual power $W_{\text {el, tot }}^{\text {act }}$ based on measurements from the operation can also be seen in the figure.

The optimal power is smaller than the predicted power, and the actual power from measurements for all steady states. As can be seen from Fig. 4, the predicted power estimated from the model differs from the actual power from measurements and the prediction error in some cases is significant, for example in steady state 27 where the actual power from measurements is almost the same as the optimal power. It is important to note that the power measurement includes inherent uncertainty which influences the estimation of the optimal power and predicted power from the data-driven models.

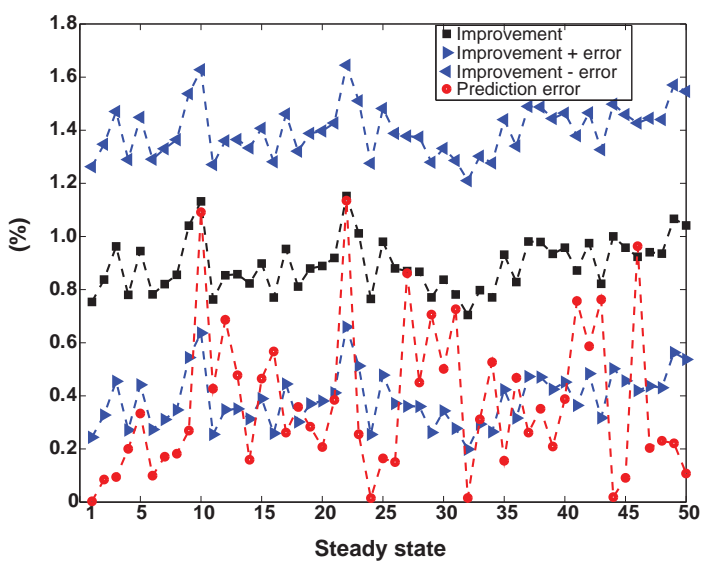

Fig. 5. Uncertainty in the metric of improvement of the operation when the RTO approach is used, and prediction error.
A metric to examine the improvement of the operation after the application of the RTO is the difference between optimal power from the model $W_{e l, t o t}^{\text {opt }} \pm \Delta W_{\text {el, tot }}$ and power from the model $W_{e l, t o t}^{\text {pred }} \pm \Delta W_{\text {el,tot }}$. The error of the metric is similarly calculated as in Eq. (6) with the root of the summation of the quadratic errors of each quantity.

Figure 5 shows the percentage of the improvement of the operation, i.e. $\left(W_{\text {el,tot }}^{\text {pred }}-W_{\text {el,tot }}^{\text {opt }}\right) / W_{\text {el,tot }}^{\text {pred }} \cdot 100 \%$ and the improvement with its uncertainty. The absolute improvement is not a large number in this example as paper [1] mentioned that one of the three compressors was operating close to its optimal set point suggested by the RTO before the application of the RTO. This means that there was not large margin for improvement. By observing the uncertainty in the improvement of the operation, the uncertainty is relatively high for this margin of improvement, and hence high accuracy models should be used. The validation of the optimal operation with use of real time optimization with real experiments in the plant could give useful insights.

Figure 5 also shows the prediction error $\mid\left(W_{\text {el,tot }}^{a c t}-\right.$ $\left.W_{\text {el,tot }}^{\text {pred }}\right) \mid / W_{\text {el,tot }}^{a c t} \cdot 100 \%$ for each steady state which corresponds to the difference between power from model and actual power from measurement. It can be seen that the prediction error can vary from close to zero (accurate prediction) to $1 \%$ error which is relatively high for the improvement of the current example.

\section{Update of model}

The update of the model is the procedure which focuses on the estimation of new coefficients $\mathbf{b}$ of the power consumption (3) for ensuring that the model represents accurately the behaviour of the compressor. For online application, the real time optimization can use a moving window approach to use new collected data and discard the oldest stored data in order to fit the model with data representing the current behaviour of the compressor.

There is not a standard method to update the parameters and there are questions to answer such as how often to update the model, what are the values of the tuning parameters of the moving window (e.g. size of window) and how to validate the model online. It was shown that the consideration of a datawindow of fifteen days and sample size of $10 \mathrm{~s}$ [1] resulted in models with higher accuracy compared to the case of one year data window and sample size of $1 \mathrm{~min}$ [10]. Andrea et al. [9] presented an online algorithm which examines first if the model is still accurate and after this evaluation it carries out a new fitting if it is necessary. However, in their work the authors studied the case of a single-stage compressor and this method required measurements to estimate the efficiency.

The performance of data-driven models can be improved with the use of information from the personnel of the plant. Changes in the plant which influence the performance of the compressors can be reported and considered in the model development, for example, the use of a different oil, the installation of a new gear box or motor, cleaning of a compressor, increased amount of fouling, the recalibration of a sensor and the plugging of the filters of compressors during 
the spring season due to the blooming of plants. If one of sensors, for example the sensor for ambient temperature, is re-calibrated during a maintenance activity, by for instance $2 \mathrm{~K}$, then the existing model can be directly updated. One approach would be to introduce a sensor error model where an offset and slope parameter are updated based on the calibration data gathered during the maintenance.

When the prediction of a data-driven model becomes significantly different from the actual values of a predicted variable, then it should be examined if the compressor or filter needs to be cleaned, or the model has to be updated. This example along with the example of the re-calibration of a sensor shows that the update of the models allows for incorporating knowledge and does not rely only on statistical approaches. Thus, this procedure for developing a datadriven model including information from the plant can be considered as "hybrid", even if the model itself is empirical.

\section{Validity domain}

The model used in Eq. (3) is generated based on data sets of process variables including inlet temperature, inlet pressure, outlet pressure and mass flow rate. These data sets are related to the input of the data-driven model which estimates the output, that is power consumption of the motor. The input data sets define the domain which allows the model to predict the power with an error. This domain is known as regression domain and predictions outside this window can give results with very low accuracy because of extrapolation. The reason for this is that the data-driven model is purely mathematical without including any physical expression which would give trends beyond the boundaries of the regression domain.

Considering a data-driven model with four inputs, the regression domain is 4-D polytope. Figure 6 shows the data sets of outlet pressure and mass flow rate of compressor $i 1$ for the first 3500 steady states of 15 days estimated offline [1]. The figure shows the time development of the regression domain of outlet pressure-mass flow rate for different peri-

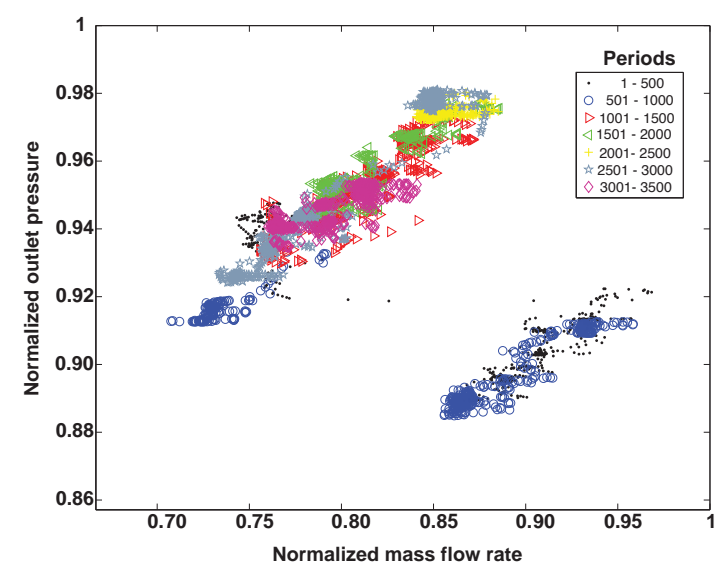

Fig. 6. Mass flow and outlet pressure data sets of compressor $i 1$ for different periods. ods. The periods are defined for different ranges of steadystates. The first observation is that this compressor worked in two separate regions where all the observed operative points define these two regions. This means that the validity of the data-driven model of compressor $i 1$ lies within these two regions. This implies that this restriction will influence the optimization as the optimization search space is defined by these two regions.

The second observation is that the compressor operated fully in the right hand region and partially in the left hand region within the first 1000 steady states. Consequently, after these 1000 steady-states the left hand region was fully developed and the operators did not operate at the right hand region in the next 2500 steady states. This means that the regression domain of the data-driven model changes over time. Considering the fact that a method for updating the model discards oldest data, then it is possible after some time the data-driven model will not have any information about the right hand region and the search space of the optimization would be limited.

Figure 7 shows period A defined as the period starting from steady state 150 until steady state 850 related to the blue data collected within this period for all the compressors. The red data were collected in period B defined from steady state 850 until steady state 5500 . The figure shows a rectangular regression domain and a convex hull of all steady states of each compressor. Here, it can be seen that the rectangular domain involves regions which do not involve any observation. The tight constraints of the convex hull prevent the model from taking these values, and therefore the reliability of the models is expected to increase as has been discussed in [11].

However, in all the cases of the convex hull of the compressors there are internal local regions which do not involve measurements. This means that the prediction in this area may not be reliable, however the optimization results may lie in this region as the constraints of the optimization model are derived from the data-driven models. The accuracy of the models probably would increase in the case of considering a conjunction of smaller convex hulls instead of one big convex hull so as to include only the observed data but this has to be studied. Finally, the convex hulls have to be developed in a 4-D fashion when four inputs are considered. Figure 7 reveals that the compressors have dissimilar boundaries in both cases of convex hull and rectangular domain for period A and B or for both periods considered. Therefore, the search space of the optimization of each model is restricted due to the restrictions of the other models.

\section{DISCUSSIONS AND CONCLUSIONS}

The paper presented the practical challenges of the application of a data-driven real time optimization to an air compressor station of an air separation plant. It was shown that the data-driven models influence the output of the optimization because of their prediction error. The improvement of the operation after the application of the RTO is determined from the comparison between the optimized power and the 

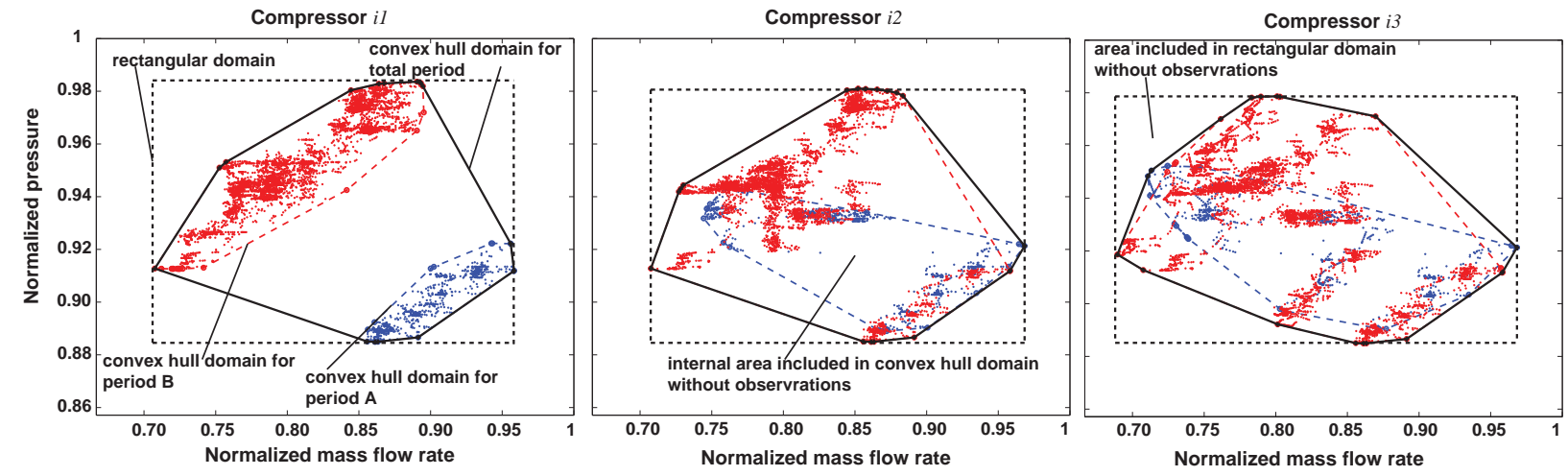

Fig. 7. Data sets and validity domains of the three compressors.

predicted power estimated using the same data-driven models based on the set points from historical data. There are errors in both quantities and the actual savings cannot be quantified accurately when the uncertainty in the models is significant.

The installation of new accurate instrumentation should benefit the model building because (a) the uncertainty in the process variables which are employed from the data-driven models should be low, (b) data reconciliation methods can be used for providing more reliable data, and (c) data-driven models can use basic thermodynamic equations to model physical phenomena, thus to achieve a hybrid model. The hybrid model is expected to be more accurate and more reliable in the prediction compared to a purely data-driven model in regions when there are limited observed data. However, the installation of new instrumentation needs to be justified by safety conditions and improved economics. For example considering Fig. 4, the management of the plant could ask the question, how much benefit could the RTO gain per year. The challenge here is that the accurate account for savings requires reliable and enough installed sensors.

According to industrial knowledge and experience, there are more sensors installed than actually displayed in the DCS, even fewer measurements are recorded in the process data historian which is the primary source of information for engineers for developing models. Hence, there are two options for improving the accuracy of the models, (a) to purchase and install new accurate sensors and (b) to connect existing sensors to the DCS and the historian system. The former case could account for several thousand euros investment and organisational effort for re-evaluating safety conditions.

The use of past and recent routine-operation data limits the optimization search space and this strategy can also lead to unreliable predictions when the predictions are related to internal regions of the regression domain with few or no observed data. Online or frequent offline plant tests can provide data to cover a larger regression domain. This can increase the accuracy of the results and increase the optimization search space.

\section{ACKNOWLEDGMENT}

Financial support from the Marie Curie FP7-ITN project "Energy savings from smart operation of electrical, process and mechanical equipment - ENERGY SMARTOPS", Contract No: PITN-GA-2010-264940 is gratefully acknowledged. The support from EPSRC Impact Acceleration Account - Imperial College London 2012, Grant EP/K503733/1 is also acknowledged. The authors would like to thank BASF SE for providing a case study and technical support.

\section{REFERENCES}

[1] D.P. Xenos, M. Cicciotti,. G.M. Kopanos, A.E.F Bouaswaig, O. Kahrs, R. Martinez-Botas and N.F. Thornhill, "Optimization of a network of compressors in parallel: Real Time Optimization (RTO) of compressors in chemical plants - An industrial case study", Applied Energy 144, pp. 51-63, 2015.

[2] I. -S. Han, C. Han and C. -B. Chung, "Optimisation of the air-and gassupply network of a chemical plant", Chemical Engineering Research and Design, Trans IChemE 82 (A10), pp. 1337-1343, 2004.

[3] F. Paparella, L. Domínguez, A. Cortinovis, M. Mercangöz, D. Pareschi and S. Bittanti, "Load sharing optimization of parallel compressors," European Control Conference (ECC), July, Zürich, Switzerland, 2013.

[4] J. Mahmoudimehr and S. Sanaye, "Minimization of Fuel Consumption of Natural Gas Compressor Stations with Similar and Dissimilar Turbo-Compressor Units, Journal of Energy Engineering 140 (1), 04013001-1:9.

[5] B. G. Lipták, "Instrument Engineer's Handbook, Process Control and Optimization", Taylor \& Francis Group 2, 4th Edition, Florida, USA, pp. 1763-1792, 2006

[6] R. Milum, Multi-compressor capacity optimization, 7th Pipeline Technology Conference 2012, 28-30 March, Hannover, Germany, 2012.

[7] R. Kurz, K. Brun, "Fouling mechanisms in axial compressors", Journal of Engineering for Gas Turbines and Power 134, pp. 032401-1:9, 2012.

[8] Siemens, Compressor Automation (SCAUT), available at: energy.siemens.com/hq/en/compression-expansion/compressorautomation.htm, accessed at 12 Oct. 2015.

[9] A. Cortinovis, M. Zovadelli, M. Mercangöz, D. Pareschi, A. De Marco and S. Bittanti, "Online adaption of performance maps for centrifugal gas compressors", European Control Conference (ECC), July 15-17, Linz, Austria, 2015.

[10] D.P. Xenos, M. Cicciotti, A.E.F Bouaswaig, N.F. Thornhill and R. Martinez-Botas, "Modeling and optimization of industrial centrifugal compressor stations employing data-driven methods", GT2014-25089, Proceedings of ASME Turbo Expo 2014, June 16-20, Düsseldorf, Germany, 2014

[11] O. Kahrs and W. Marquardt, The validity domain of hybrid models and its application in process optimization, Chemical Engineering and Processing 46, pp. 1054-1066. 